\title{
EGF Activates Autocrine TGF $\alpha$ to Induce Prolonged EGF Receptor Signaling and Hepatocyte Proliferation
}

\author{
ShuZheng Liu a, b Lene Wierød ${ }^{\mathrm{b}}$ Ellen Skarpen ${ }^{\mathrm{b}, \mathrm{c}}$ Helga Grøsvik ${ }^{\mathrm{b}}$ GuangCai Duan $^{\mathrm{a}}$ \\ Henrik S. Huitfeldt ${ }^{b}$
}

\begin{abstract}
aDepartment of Epidemiology, College of Public Health, Zhengzhou University, Zhengzhou, Henan, China; 'bepartment of Pathology, Oslo University Hospital, University of Oslo, Oslo, Norway; 'Department of Biochemistry, Oslo University Hospital, Oslo, Norway
\end{abstract}

\section{Key Words}

EGF receptor - Autocrine TGF $\alpha$ - Sustained Erk1/2 activation - Cyclin D1 •DNA synthesis • Primary hepatocytes

\begin{abstract}
Background/Aims: EGF receptor is a main participant in the regulation of liver regeneration. In primary hepatocyte cultures, EGF or TGF $\alpha$ binding to EGF receptor activates Erk1/2 and PI3K pathways, induces cyclin D1 and thus initiates DNA synthesis. We have explored mechanisms by which prolonged EGF receptor activation induces hepatocyte proliferation. Methods: EGF receptor activation, as well as Erk1/2 and PI3K signaling were explored in EGF-stimulated primary hepatocyte cultures by Western blotting and immunocytochemistry. TGF $\alpha$ release to the medium was quantified by ELISA. Effects of a neutralizing antibody to TGF $\alpha$ on EGF receptor signaling and proliferation were explored. Results: Inhibitors of PI3K or Erk1/2 inhibited cyclin D1 expression and $\mathrm{G}_{1}$ progression when added 12 hours after EGF stimulation, whereas depletion of EGF from the medium at this time point did not. ELISA demonstrated that EGF induced TGF $\alpha$ release to the medium. Cyclin D1 induction and cellular proliferation were efficiently inhibited when a neutralizing antibody to TGF $\alpha$ was added to the medium. This also occurred when the antibody was added 12 hours after EGF stimulation. Conclusion: Sustained EGF receptor activity and signaling through both Erk1/2 and PI3K pathways were necessary for proliferation. This was achieved by EGF activation of autocrine TGF $\alpha$.
\end{abstract}

Copyright $\odot 2013$ S. Karger AG, Basel 


\section{Introduction}

Liver regeneration requires that priming factors (TNF $\alpha$, IL6) recruit cells into the cell cycle, whereas growth factors (HGF, EGF, TGF $\alpha$ ) promote cell cycle progression through a midlate $G_{1}$ restriction point (R point) $[1,2]$. To gain insight into mechanisms of liver regeneration and carcinogenesis, these mechanisms have been intensely studied in primary hepatocyte cultures. In such cultures, priming and cyclin E induction are achieved at hepatocyte isolation and plating, whereas cyclin D1 expression is strictly regulated by EGF, TGF $\alpha$ and HGF. These growth factors bind to and activate their respective receptors; EGF receptor or c-Met [3, 4]. D-type cyclins appear to act as intracellular "sensors" of extracellular stimuli that promote proliferation [5, 6]. EGF receptor and c-Met both activate the phosphatidyl inositol 3-kinase (PI3K) and the Extracellular signal-regulated kinase (Erk1/2) signaling pathways, which are required for hepatocyte cyclin D1 induction in response to mitogens [7, 8]. Prolonged activation and nuclear translocation of Erk1/2 are necessary for induction of proliferation [9]. Several studies have shown that sustained Erk1/2 activity is necessary for cyclin D1 induction and proliferation $[8,10]$. In primary hepatocytes, EGF and TGF $\alpha$ induce a sustained elevation of ERK activity, lasting for at least 24 hours [11].

EGF is not produced in the liver, but is made in kidneys, guts, and salivary glands. Several studies have suggested the participation of endocrine EGF in the regulation of liver regeneration [12]. The demonstration of TGF $\alpha$ induction in hepatocytes of regenerating livers, as well as in vitro studies of TGF $\alpha$ effects on hepatocyte proliferation, pointed towards an autocrine TGF $\alpha$ mechanism being important for liver regeneration [13]. Also, it has been demonstrated that several cytokines and growth factors, including prostaglandin E2, TNF $\alpha$ and HGF, may transactivate EGF receptor through activation of autocrine TGF $\alpha$ signaling [14$16]$.

We have investigated mechanisms underlying sustained EGF receptor signaling in cultured hepatocytes, in particular in the period after EGF receptor has been internalized and down-regulated. Results showed that maintenance of sustained EGF receptor signaling and proliferation of hepatocytes by EGF was dependent upon autocrine TGF $\alpha$ signaling.

\section{Materials and Methods}

\section{Materials}

Williams' medium E, Dulbecco's modified Eagle's medium, penicillin and streptomycin were from Gibco (Grand Island, NY, USA). Collagenase (C-0130), collagen (C-7661), dexamethasone (D-4902), EGF (E-1257), and insulin (I-6634) were obtained from Sigma-Aldrich (St Louis, MO, USA). PD153035 was purchased from Calbiochem (La Jolla, CA, USA), PD184352 from Axon Biochemicals (Groningen, Netherlands) and LY294002 from Promega Corporation (Fitchburg, WI, USA). TGF $\alpha$ was obtained from Bachem (Weil am Rhein, Germany). ${ }^{3} \mathrm{H}$-thymidine was obtained from GE Healthcare (London, UK).

The following antibodies were used: Sheep anti-EGF receptor from Fitzgerald (Acton, MA, USA), rabbit anti-pThr202/pTyr204 Erk1/2, rabbit anti-Akt, and rabbit anti-pSer473Akt from Cell Signalling Technology (Beverly, MA, USA), rabbit anti-Erk1, mouse anti-cyclin E, rabbit anti-Cdk2, and rabbit anti-Cdk4 from Santa Cruz Biotechnology (Santa Cruz, CA), rabbit anti-cyclin D1 from Upstate Biotechnology (Lake Placid, NY, USA), mouse anti- $\beta$-tubulin from Sigma-Aldrich, St. Louis, MO, USA, mouse monoclonal anti-TGF $\alpha$ from Calbiochem (Calbiochem/EMD Chemicals, Inc., Merck KGaA, Darmstadt, Germany) [17], and rabbit antipTyr1173 EGF receptor [18]. Sub- and isotype-matched control immunoglobulin for anti-TGF $\alpha$ was from , Sigma Aldrich, St. Louis, MO, USA (murine IgG1, kappa; clone number MOPC 21).

\section{Cell isolation and culture}

Young adult male Wistar rats (Møllergård and Bomhoff, Odense, Denmark), weighing 200- 220 g, were kept on a 12-h light: dark cycle and were fed ad libitum. Hepatocytes were isolated in a two-step in vitro version of the collagenase perfusion technique with modifications [19-21]. Cells were seeded at a density of 20000 cells $/ \mathrm{cm} 2$. Serum-free culture medium consisted of a 1:1 combination of Williams Medium 
Liu et al.: EGF Induces Hepatocyte Proliferation Through Autocrine TGF $\alpha$

E and Dulbecco's modified Eagle's medium, with a final glucose concentration of $8.4 \mathrm{mM}$. Medium was supplemented with penicillin $(67 \mu \mathrm{g} / \mathrm{ml})$, streptomycin $(100 \mu \mathrm{g} / \mathrm{ml})$, collagen $(3 \mu \mathrm{g} / \mathrm{ml})$, dexamethasone $(25 \mathrm{nM})$ and insulin $(100 \mathrm{nM})$. The cells were plated with $5 \%$ fetal calf serum (Sigma-Aldrich, St. Louis, MO, USA) during the first three hours, and thereafter maintained in medium without serum in a $5 \% \mathrm{CO}_{2}$ atmosphere at $37^{\circ} \mathrm{C}$. EGF $(10 \mathrm{nM})$ were added 20 hours after cell plating. Anti-TGF $\alpha$ was added to the media to a final concentration of $0.15 \mu \mathrm{g} / \mathrm{ml}$ unless otherwise stated. EGF receptor inhibitor (PD153035) was used at a concentration of $1 \mu \mathrm{M}$, Mek inhibitor (PD184352) at $5 \mu \mathrm{M}$, and PI3K inhibitor (LY294002) at $10 \mu \mathrm{M}$. In experiments where these inhibitors were used, control and EGF-exposed hepatocytes were treated with DMSO at the same concentration (0.1\%).

The neutralizing antibody to anti-TGF $\alpha$ was controlled for cross-reactivity. EGF (300nM) was incubated with anti-TGF $\alpha(15 \mathrm{ug} / \mathrm{ml})$ or control antibody (15 ug/ml), in $100 \mathrm{ul} \mathrm{medium} \mathrm{for} 1$ hour before addition to hepatocyte cultures (final concentration of EGF 10nM, final concentration of antibodies $0.5 \mathrm{ug} /$ $\mathrm{ml}$ ). Cells were harvested after 10 minutes, and subjected to Western blotting for pTyr1173 EGF receptor and pThr202/pTyr204 Erk1/2.

\section{Western immunoblot analysis}

Cultured hepatocytes were harvested and processed for Western immunoblot as previously described $[20,21]$. Briefly, cells were lysed and sonicated in a Tris-lysis buffer, (pH 7.4, with $60 \mathrm{~mm}$ Tris- $\mathrm{HCl}, 10 \%$ glycerol, 3\% sodium dodecyl sulfate, $1 \mathrm{~m}$ EDTA, $0.2 \mathrm{~mm}$ AEBSF, $20 \mu \mathrm{m}$ leupeptin, 200 units $/ \mathrm{mL}$ aprotinin, $65 \mu \mathrm{m}$ sodium orthovanadate and $10 \mathrm{~mm} \beta$-glycerophosphate). Protein concentrations of all samples were measured by DC protein assay (Bio-Rad, Hercules, CA, USA), and protein concentrations adjusted to equal concentrations in the lysis buffer containing $5 \% \beta$-mercaptoethanol and $0.0025 \%$ bromophenol blue. Protein samples were boiled for $4 \mathrm{~min}$ and were stored at $-20{ }^{\circ} \mathrm{C}$. Proteins were separated by SDSPAGE and were electrotransferred to nitrocellulose membranes (GE Healthcare, Little Chalfont, United Kingdom) for subsequent protein detection. Filters were blocked in Tris-buffered saline containing 5\% fat-free dry milk, and were incubated overnight at $4{ }^{\circ} \mathrm{C}$ with primary antibodies diluted in Tris-buffered saline containing $1 \%$ fat-free dry milk. After washing, filters were incubated with horseradish peroxidase (HRP)-conjugated goat anti-rabbit IgG, donkey anti-mouse IgG, or donkey anti-sheep IgG from Sigma Aldrich at room-temperature for $90 \mathrm{~min}$. The detection of immunoreactivity was achieved by incubation in SuperSignal WestDura Extended Duration Substrate (Thermo Scientific, Walthan, MA), followed by signal detection and densitometry with a Kodak Image station 4000R equipped with Kodak Molecular Imaging 4.0 software.(Eastman Kodak Company, Rochester, NY, USA). Mean and SEM values for 3 experiments were calculated after normalization. For EGF receptor, Erk1, Erk2, Akt, pSer473Akt, and cyclin E densitometric measurements from the initial control sample (without EGF) were set to 100\%. For pY1173 EGF receptor, pThr202/pTyr204 Erk1 and Erk2 measurements from 10 minutes EGF stimulation were set to 100\%. For cyclin D, measurements from the 30 hours time point after EGF stimulation was set to $100 \%$.

Immunoblotting for $\beta$-tubulin was used as loading controls. In these and previous investigations we experienced increasing expression of $\beta$-tubulin during the culturing period, in particular after EGF stimulation. This has also been observed for other housekeeping gene products. Identification of appropriate and robust loading controls for mRNA and protein investigations may be problematic [22]. Increasing levels of cell signaling proteins during the culturing period has also been described in primary hepatocyte cultures [23]. For these reasons, densitometry data are given both as normalized protein levels, and as phosphoprotein to protein or protein to $\beta$-tubulin ratios.

\section{Immunocytochemistry and confocal laser scan microscopy}

Immunofluorescence staining was performed as previously described [18, 20, 21]. Briefly, hepatocyte cultures were fixed in 70\% ethanol or 4\% PBS-buffered paraformaldehyde for 10 minutes. The latter was followed by a 10 minutes wash in PBS containing $0.02 \%$ TRITON X100. Thereafter, cells were washed for 10 minutes in PBS, rinsed in deionized $\mathrm{H}_{2} \mathrm{O}$, and dried. Cells were incubated overnight with combinations of primary antibodies, as indicated in Results. All antibodies were diluted in in PBS containing $1 \%$ bovine serum albumin and $0.01 \%$ thimerosal. After washing procedures (2X10 minutes in PBS followed by a dip in deionized $\mathrm{H}_{2} \mathrm{O}$ and drying), immunobinding was detected with appropriate combinations of species specific, fluorochrome-conjugated donkey antisera to IgG (Multiple Labeling series; Jackson ImmunoResearch, West Grove, PA, USA). After a new washing procedure, the cells were mounted in "Dako fluorescent mounting 
Liu et al.: EGF Induces Hepatocyte Proliferation Through Autocrine TGF $\alpha$

medium" (Dako, Glostrup, Denmark). As controls for cross-reactivity, the primary antibodies were substituted with antibodies to irrelevant antigens at appropriate dilutions. The immunostained cells were examined with an Olympus FV1000 confocal microscope (Olympus Corporation, Tokyo, Japan). A UPlanSApo $60 \mathrm{x} / 1.35$ oil objective was used. Sequential scanning was used for imaging.

Measurement of DNA synthesis

For thymidine incorporation cells were cultured in six-well plates and grown as described above. ${ }^{3} \mathrm{H}$-thymidine $(1 \mathrm{uCi} / \mathrm{ml})$ was added to the cultures 24 hours after EGF stimulation, and the cells harvested 32 hours thereafter. DNA synthesis was assessed by determining the amount of radioactivity incorporated into DNA per mg protein. The cellular material was precipitated with $2 \mathrm{ml} 5 \%$ trichloroacetic acid for $2 \times 10$ $\mathrm{min}$. The acid-precipitated material was dissolved in $0.3 \mathrm{ml} 1 \mathrm{M} \mathrm{KOH}$, followed by liquid scintillation counting. Protein was measured as earlier described. Mean and SEM values of at least 3 experiments were calculated after normalization of results from separate experiments, where the CPM/protein ratios of hepatocytes exposed to EGF alone were set to $100 \%$

ELISA

Medium was collected, supplemented with $0.2 \mathrm{mM}$ AEBSF, $20 \mu \mathrm{M}$ leupeptin, 200 units/ml aprotinin, 65 $\mu \mathrm{M}$ sodium orthovanadate and $10 \mathrm{mM} \beta$-glycerophosphate.and assayed for TGF $\alpha$ with a quantitative ELISA kit (Calbiochem/EMD Chemicals, Inc., Merck KGaA, Darmstadt, Germany) according to the manufacturer's protocol. Samples were quantified against a standard curve using known amounts of lyophilized control TGF $\alpha$ peptide reconstituted in deionized $\mathrm{H}_{2} \mathrm{O}$ and then serially diluted in assay buffer to concentrations of $63,32,16$ and $8 \mathrm{pg} / \mathrm{ml}$. All assays were carried out in duplicate, and repeated at least 3 times.

\section{Statistical analysis}

Quantitative data are presented as mean and standard errors of the mean (SEM). Mean thymidine incorporations were calculated by normalizing results from single experiments, setting thymidine incorporation of EGF-stimulated hepatocytes to $100 \%$. Statistically significant differences were analyzed with Student's t-test. All calculations were made with GraphPad Prism version 5.0. A p-value of $<0.05$ was considered significant compared to its control, marked with *.

\section{Results}

\section{EGF induced prolonged Erk1/2 activation}

EGF receptor activation has been shown to stimulate Erk1/2 and PI3K/Akt signaling pathways. Ligand binding induces receptor activation, internalization and ultimately degradation, thus attenuating the receptor signaling [24]. Prolonged Erk1/2 activation has been observed to be necessary for growth factor induced proliferation [9]. In primary hepatocytes it has been demonstrated that sustained Erk1/2 activity during the whole $\mathrm{G}_{1}$ phase is necessary for S phase entry $[8,10]$. Several studies have also demonstrated the necessity for PI3K activation for induction of cyclin D1, and thus hepatocyte proliferation [7]. To further investigate the activation of these two signaling pathways, EGF receptor, Erk1/2 and Akt phosphorylation following EGF stimulation was studied. As shown in Fig.1A and $B$, when the medium was supplemented with EGF, the EGF receptor immediately became phosphorylated. During the following 6-12 hours total EGF receptor expression diminished, and remained low for the remaining period. From 18 hours after EGF stimulation, protein levels of Erk 1 and 2 were induced. Erk1 and 2 became phosphorylated simultaneously with EGF receptor. During the following 6-12 hours Erk1 showed declining phosphorylation levels paralleling EGF receptor phosphorylation, whereas Erk2 showed persistent phosphorylation, also at time points when EGF receptor was maximally down-regulated (12-24 hours). Increased levels of Erk1/2 phosphorylation were noted at 30 hours. Akt showed phosphorylation prior to EGF stimulation, and this was increased at 10 and 30 minutes following EGF stimulation. Thereafter, Akt showed declining phosphorylation levels, reaching lower than initial levels 6-18 hours after EGF stimulation. 


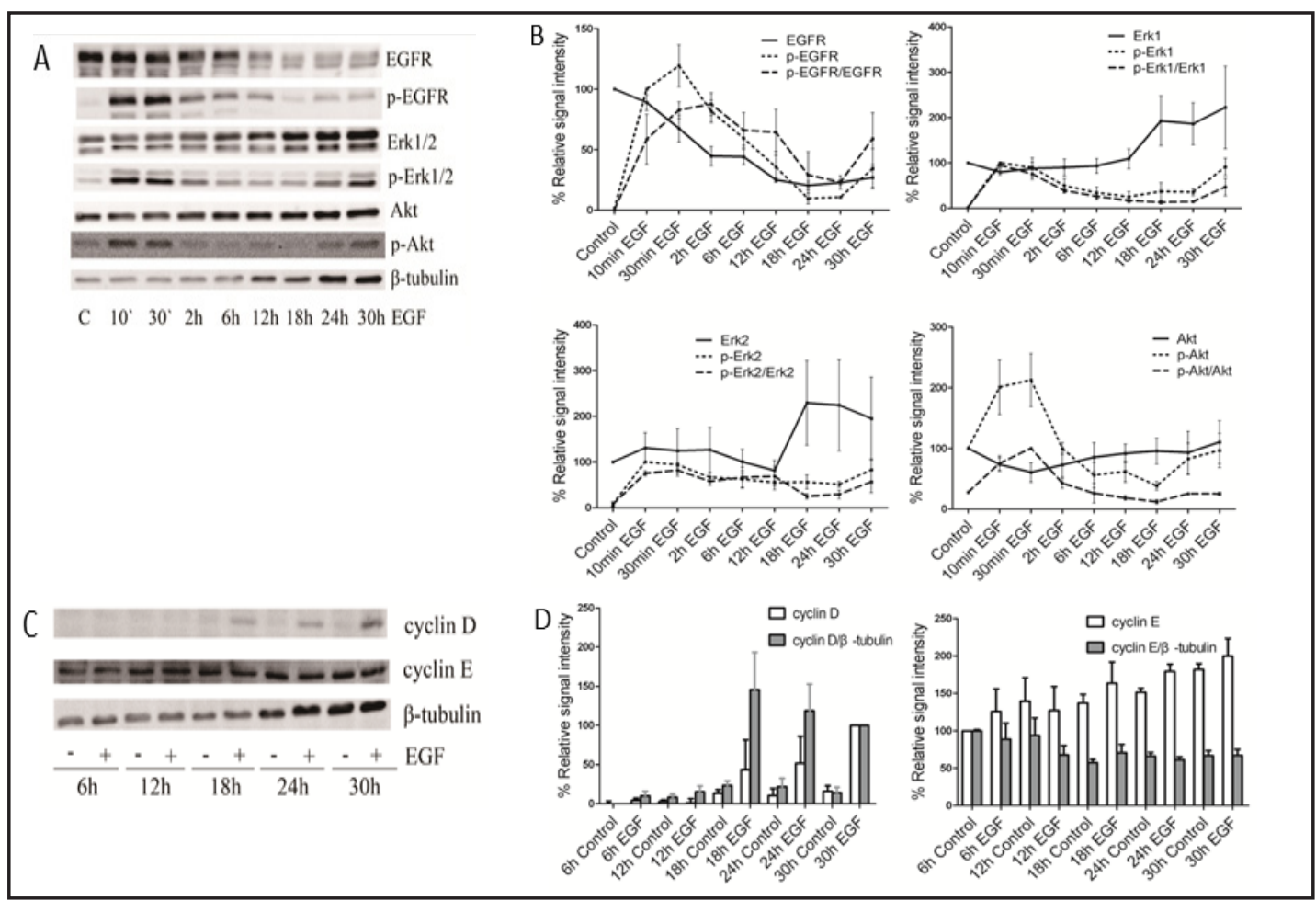

Fig. 1. EGF receptor signaling following EGF stimulation. (A) Primary hepatocytes were stimulated with EGF and harvested at different time points (10 min.-30 hrs) thereafter. Western immunoblot analyses of EGF-induced signaling Western immunoblots were performed on lysates with antibodies to EGF receptor, pTyr1173 EGF receptor, Erk1/2, pThr202/pTyr204 Erk1/2, Akt, pSer473 Akt, and $\beta$-tubulin. (B) Densitometry of EGF receptor signaling proteins following EGF stimulation Densitometry data for EGF receptor, Erk1, Erk2, and Akt. Data are displayed as normalized protein levels, normalized phosphoprotein levels, and phosphoprotein to protein ratios for each signaling protein ( $n=3$, error bar: SEM). (C) Western immunoblot analyses for $G_{1}$ cyclins. Western immunoblots for cyclin $D$ and cyclin $E$ were performed on lysates from EGF stimulated hepatocytes and unstimulated controls harvested at different time points (6$30 \mathrm{hrs}$ ) after growth factor stimulation. (D) Densitometry of $\mathrm{G}_{1}$ cyclins. Western immunoblots for cyclin D and cyclin E were subjected to densitometry. Data are displayed as normalized protein level, and protein to $\beta$-tubulin level ratios $(n=3$, error bar: SEM).

Having demonstrated that EGF receptor activity and signaling were present even after EGF receptor down-regulation; we wanted to identify which cell cycle regulators were affected by the late phase of Erk1/2 and PI3K activity; i.e. after growth factor receptor down regulation. Growth factor activation of $\mathrm{Cdk} 2$ and $\mathrm{Cdk} 4$ drives the cell cycle progression until a checkpoint in late $G_{1}$. In primary hepatocytes, cyclin $E$ appears to be induced by cell isolation or plating and is thereafter unaffected by growth factor addition [4]. Induction of cyclin D1 through Erk1/2 and PI3K signaling appears to be the main, growth factor regulated event leading to $\mathrm{pRb}$ hyperphosphorylation $[3,4]$. To correlate $G_{1}$ cyclin induction with EGF receptor activation and signaling, we studied the time course of cyclin E and D1 expression following EGF stimulation. As also observed previously, cyclin D1 was induced from 18 hours after EGF stimulation, and remained high through the period where EGF receptor activation was very low (Fig. 1C and D) [4]. Cyclin E was present and unaffected by EGF stimulation through the studied period.

\section{Sustained PI3K/Erk1/2 activity was necessary for proliferation}

To explore the necessity for sustained EGF receptor activity and signaling, we investigated hepatocyte EGF-induced cell cycle progression when the EGF receptor kinase, Mek, or PI3K were inhibited by the addition of DMSO (vehicle), PD153035, PD184352, 
Fig. 2. Sustained PI3K/Erk1/2 activity was necessary for proliferation. (A) Effects of late EGF receptor signaling inhibition on hepatocyte proliferation. Primary cultures of hepatocytes were exposed to EGF receptor kinase inhibitor, MEK inhibitor, or PI3K inhibitor $12 \mathrm{~h}$ after EGF stimulation. The cells were harvested $30 \mathrm{~h}$ after EGF stimulation. Tritiated thymidine incorporation was measured by scintillation counting. The results represent an average

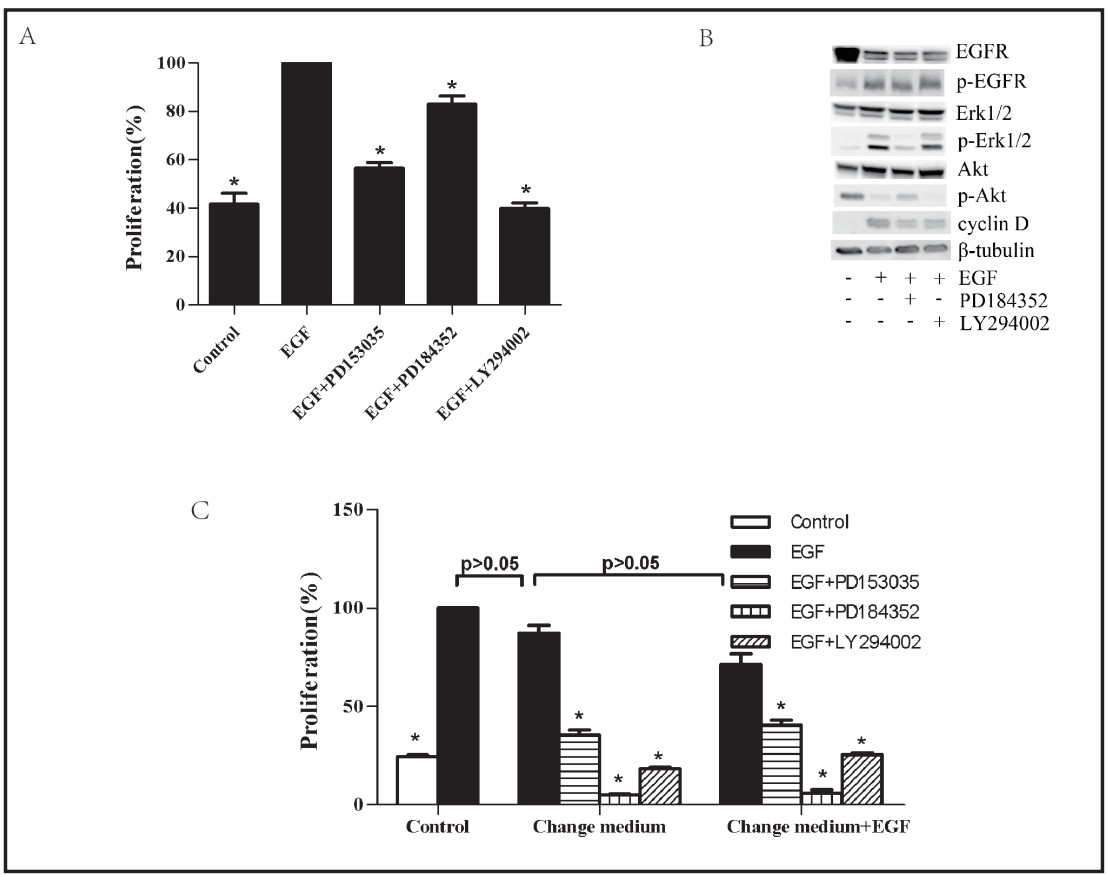
of three separate experiments (SEM; $n=3$ ). *) significant versus EGF, $p<0.05$. (B) Western blot analyses for EGF receptor signaling proteins following late signaling inhibition. Primary hepatocyte cultures, unstimulated or exposed to EGF and harvested 24 hours later (lane 1 and 2), were analyzed by Western blotting for EGF receptor, pTyr1173 EGF receptor, Erk1/2, pThr202/pTyr204 Erk1/2, Akt, pSer473 Akt, and cyclin D. Immunoblotting for $\beta$-tubulin was used as a loading control. MEK inhibitor or PI3K inhibitor were added $12 \mathrm{~h}$ after EGF stimulation in indicated samples (lane 3 and 4). (C) Effects of late medium change on EGFstimulated hepatocyte proliferation. Primary cultures of hepatocytes were left untreated or stimulated with EGF. In some samples, the medium was substituted 12 hours after growth factor stimulation with medium without EGF, with medium without EGF but with either EGF receptor inhibitor, MEK inhibitor, or PI3K inhibitor added, with medium with freshly added EGF, or the same medium containing either EGF receptor inhibitor, MEK inhibitor, or PI3K inhibitor. Proliferation was determined by tritiated thymidine incorporation. The results represent an average of three separate experiments (SEM; $n=3$ ). ${ }^{*}$ ) significant versus EGF of each group, $\mathrm{p}<0.05$.

or LY294002, respectively, 12 hours after growth factor stimulation. Tritiated thymidine was added 24 hours after EGF, and the cells were harvested 6 hours thereafter. Results showed that inhibition of EGF receptor kinase, Mek or PI3K activities reduced hepatocyte proliferation, demonstrating that EGF receptor activity and signaling were necessary even after EGF receptor down-regulation (Fig. 2A).

Western blotting was performed on cells harvested 24 hours after EGF stimulation of hepatocytes. DMSO (vehicle), MEK inhibitor (PD184352), or PI3 kinase inhibitor (LY294002) were added to the medium 12 hours after EGF stimulation. Results showed that increased phosphorylation of EGF receptor and Erk1/2 was still detectable at this time point, whereas Akt phosphorylation was lower in EGF-stimulated cells than in unstimulated controls. Cyclin D1 expression was inhibited by both Mek and PI3 kinase inhibitors (Fig. 2B).

To explore whether the prolonged EGF receptor activity and signaling were EGF dependent, proliferation was investigated in EGF stimulated cells. Twelve hours after EGF stimulation, a medium change was performed. Medium was substituted with medium without growth factors, with medium without growth factors but containing EGF receptor kinase inhibitor (PD153035), MEK inhibitor (PD184352), or PI3K inhibitor (LY294002), or with medium containing freshly added EGF $(10 \mathrm{nM})$. Results showed that substitution with a growth factor-free medium had little effect on hepatocyte proliferation, whereas substitution 
Fig. 3. EGF stimulation activated A autocrine TGF $\alpha$ signaling. (A) ELISA quantitation of $\mathrm{TGF} \alpha$ release to the medium. Primary hepatocytes were left untreated (labeled Control) or stimulated with B EGF (labeled EGF). Medium was collected 24 hours after growth factor stimulation, and TGF $\alpha$ concentration in the medium measured by ELISA. Results are presented as mean \pm SEM $(n=3)$ *) significant versus Control, $\mathrm{p}<0.05$. (B) Dose response effects of neutralizing anti-TGF $\alpha$ effects on EGF-induced proli-

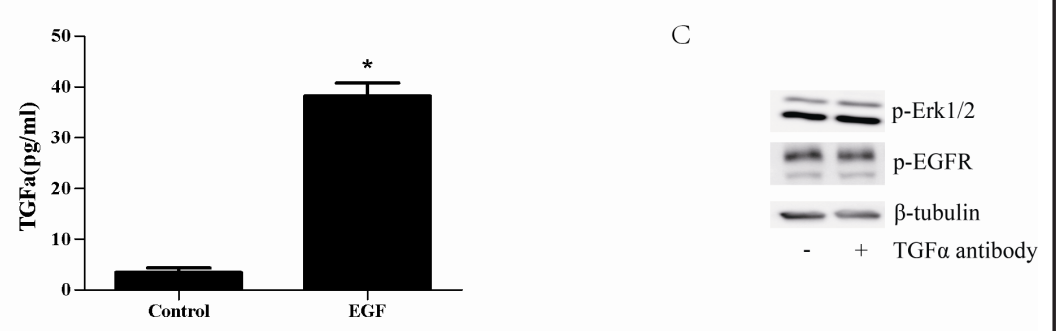

feration. Primary cultures of hepatocytes were exposed to control antibody (0.5ug/ml) or TGF $\alpha$ antibody $(0.05 \mathrm{ug} / \mathrm{ml}, 0.15 \mathrm{ug} / \mathrm{ml}, 0.5 \mathrm{ug} / \mathrm{ml}) 1 \mathrm{~h}$ prior to EGF stimulation. The cells were harvested $30 \mathrm{~h}$ after EGF stimulation, and tritiated thymidine incorporation measured. The results represent an average of three separate experiments (SEM; $n=3$ ). *) significant versus $E G F+C o n t r o l$ antibody, $p<0.05$. (C) EGF cross reactivity control for anti- TGF $\alpha$ antibody. EGF was incubated with anti-TGF $\alpha$ or control antibody for 1 hour before addition to hepatocytes. Cells were harvested after 10 minutes, and subjected to Western blotting for pTyr1173 EGF receptor and pThr202/pTyr204 Erk1/2. Blotting for $\beta$-tubulin was used as a loading control.

with the same medium containing inhibitors of EGF receptor, MEK, or PI3K substantially reduced proliferation (Fig. 2C). Thus, it appeared as proliferation was dependent on EGF receptor activity and signaling, and that this activity was not caused by EGF in the medium.

\section{EGF stimulation activated autocrine TGF $\alpha$ signaling}

Since it appeared as sustained EGF receptor signaling occurred independently of EGF binding, we speculated whether an autocrine EGF receptor ligand was activated. It has been demonstrated that $\mathrm{HGF}, \mathrm{TNF} \alpha$ and prostaglandin $\mathrm{E}(2)$ promotes proliferation by autocrine TGF $\alpha$ activation [13-15]. Thus, we hypothesized that similar mechanisms were involved in EGF induced proliferation.

First, we explored whether TGF $\alpha$ was released into the medium from EGF-stimulated hepatocytes. Hepatocytes were incubated with EGF or vehicle alone for 24 hours, and TGF $\alpha$ concentrations in the medium assayed with an ELISA kit. Results showed that TGF $\alpha$ levels in the media, albeit low, were about 10 -fold higher in EGF-stimulated samples than in controls (Fig. 3A).

We then explored whether the presence of TGF $\alpha$ was necessary for hepatocyte proliferation. Hepatocytes were incubated with or without EGF for 30 hours. One hour prior 
Fig. 4. Activation of cyclin D1 was dependent on autocrine TGF $\alpha$. (A) Anti-TGF $\alpha$ effects on sustained EGF receptor signaling and G1 cyclin induction. Primary hepatocytes were incubated with TGF $\alpha$ antibody or a control antibody for 1 hour prior to EGF stimulation, and harvested at different time points (as indicated) thereafter. Western immunoblot analyses were performed on lysates with antibodies to Erk1/2, pThr202/ pTyr204 Erk1/2, Akt, pSer473 Akt, cyclin D, and cyclin $\mathrm{E}$ The protein expression of $\beta$-tubulin was used as a loading control. A representative example of at least three experiments is shown. (B) Anti-TGF $\alpha$ effects on cyclin D/Cdk4 localization. Unstimulated (a-c) or EGF stimulated (d-i) primary hepatocytes were exposed to control antibody (a-f) or neutralizing antibody to TGF $\alpha$ (g-i) 1 hour prior to growth factor stimulation. Cells were stained by paired immunofluorescence for cyclin D (a, d, g; Alexa 488 (green) emission) in combination with Cdk4 (b, e, h, Alexa555 (red) emission). Overlays of Alexa 488 and Alexa 555 fluorescence is demonstrated in c, f, and i. Cell cultured stained by immunofluorescence were examined by confocal microscopy (60x oil immersion objective, zoom 1).

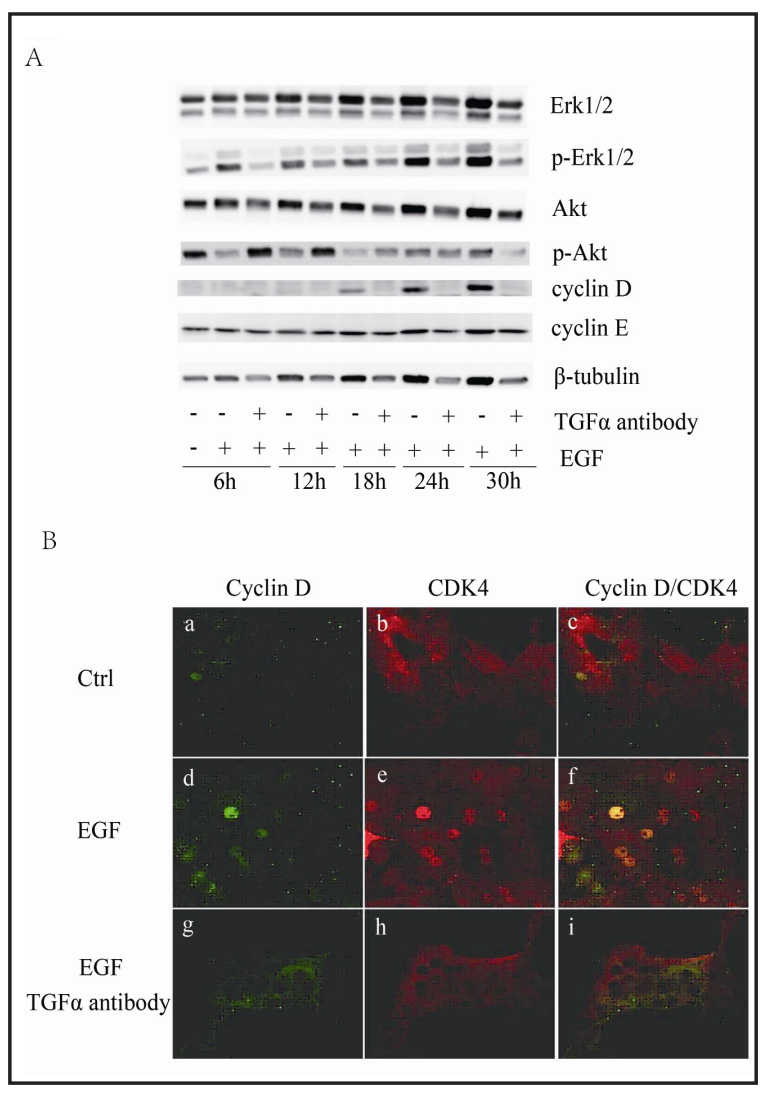

to growth factor addition, increasing concentrations of a neutralizing mouse antibody to TGF $\alpha$, or a sub- and isoform-matched control mouse antibody, were added [17]. Twenty-four hours after EGF stimulation, tritiated thymidine was added. Measurements of thymidine incorporation showed that proliferation was efficiently and dose-dependently inhibited to levels lower than in unstimulated hepatocytes at an antibody concentration of 0.5 and $0.15 \mu \mathrm{g} / \mathrm{ml}$. The latter concentration was used for further experiments (Fig. 3B).

To ensure that the effect of the neutralizing antibody was not an effect of crossreactivity with EGF, this antibody and the sub- and isoform-matched control antibody were incubated for 1 hour with the medium containing EGF, and applied to hepatocyte cultures. Cells were harvested for Western blotting 10 minutes thereafter. Western blotting for EGF receptor phosphorylation and Erk1/2 phosphorylation showed that preincubation of EGF with the neutralizing antibody did not reduce the initial, EGF-induced phosphorylations of EGF receptor or Erk1/2 (Fig. 3C).

Activation of cyclin D1 was dependent on autocrine TGFa even in the presence of EGF

We explored the effect of the neutralizing antibody to TGF $\alpha$ on sustained Erk1/2 and PI3K/Akt activation (Fig. 4A). Anti-TGF $\alpha$ or control antibody was added to the culture medium 1 hour prior to EGF stimulation. Cells were harvested 6, 12, 18, 24 and 30 hours after growth factor addition, and investigated by Western blotting. Results showed increasing levels of Erk, Akt and $\beta$-tubulin following EGF stimulation, and this was counteracted by the neutralizing anti-TGF $\alpha$. During the period from 6 to 30 hours after EGF stimulation, Erk1/2 phosphoprylation remained higher than in unstimulated hepatocytes. In EGF-stimulated hepatocytes treated with the antibody to TGF $\alpha$, Erk1/2 phosphorylation remained low during this period. Unstimulated hepatocytes displayed comparatively high Akt phosphorylation, and these levels were conspicuously reduced 6 hours after EGF stimulation. In cells treated with EGF and anti-TGF $\alpha$ antibody, Akt phosphorylation remained high at 6 and 12 hours after EGF, whereafter Akt phosphorylation became similar to EGF-stimulated controls. 
Fig. 5. Autocrine TGF $\alpha$ stimulation during sustained EGF receptor signaling was necessary for proliferation. (A) Effects of anti-TGF $\alpha$ addition during sustained EGF receptor signaling on hepatocyte proliferation. Primary cultures of hepatocytes were exposed to control antibody or TGF $\alpha$ antibody 1 hour prior to, 12 hours after, or 24 hours after EGF stimulation. Thymidine incorporation was measured. The results represent an average of three separate experiments. (SEM; $n=3$ ). *) significant versus control antibody of each group, $p<0.05$. (B) Effects of anti-TGF $\alpha$ addition during sustained EGF receptor on $\mathrm{G}_{1}$ cyclin induction. Primary hepatocyte cultures were either left unstimulated or exposed to EGF, as indicated. Twelve hours thereafter, control antibody or a neutralizing TGF $\alpha$ antibody was added, as indicated. Cells were harvested at 12, 18, 24 or 30 hours after EGF stimulation, and subjected to Western blotting for cyclin $\mathrm{D}$ and cyclin E expression. Blotting for $\beta$-tubulin was used as a loading control.

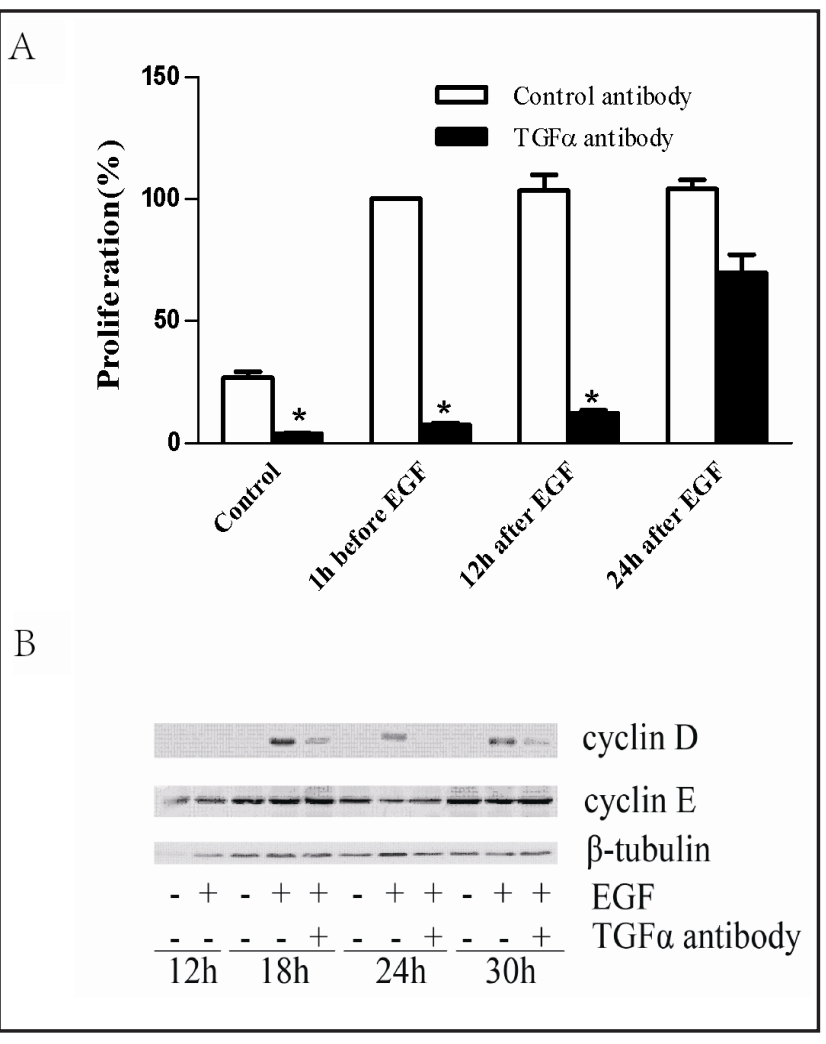

Also the expression of G-phase cyclins was explored by Western blotting. The antibody to TGF $\alpha$ did not appear to alter cyclin E expression, but completely inhibited the EGF-induced cyclin D1 induction observed from 18 hours after EGF stimulation.

Effects of the neutralizing antibody to TGF $\alpha$ were also explored by immunocytochemistry (Fig. 4B). Cells were exposed to a control antibody, or to the antibody to TGF $\alpha$, and stimulated with EGF one hour later. Twenty-four and 30 hours after EGF stimulation, cell cultures were fixed with $96 \%$ ethanol, and stained for cyclin E, cyclin D1, Cdk2, and Cdk4. We found that the neutralizing antibody to TGF $\alpha$ inhibited EGF-induced nuclear accumulation of all these cell cycle regulators (cyclin E and Cdk2 not shown).

Autocrine TGFa prolongation of EGF receptor signaling was necessary for induction of hepatocyte proliferation

To further explore whether autocrine TGF $\alpha$ stimulation contributed to the sustained Erk1/2 and Akt activity and thus to cell cycle progression, primary cultures of hepatocytes were exposed to the neutralizing TGF $\alpha$ antibody or control antibody at different time points after EGF stimulation (Fig. 5A). Tritiated thymidine was added 24 hours after EGF, and the cells were harvested for detection of thymidine incorporation at 30 hours after EGF. We found that the antibody to TGF $\alpha$ totally inhibited EGF-stimulated hepatocytes proliferation when added 1 hour before or 12 hours after the growth factor. A partial effect was found when the antibody was added 24 hours after EGF. Thus, it appeared as autocrine TGF $\alpha$ stimulation during the sustained phase of EGF receptor signaling was necessary for $S$ phase entry. Proliferation of hepatocytes that had not been stimulated with EGF was also inhibited by the antibody, indicating that endogenous, autocrine TGF $\alpha$ stimulation caused a "background" proliferation.

Effects of autocrine TGF $\alpha$ depletion 12 hours after EGF stimulation on cyclin D1 and E regulation was investigated by Western blotting. Results showed that cyclin D1 expression in this period was dependent on sustained TGF $\alpha$ stimulation, whereas cyclin E appeared unaffected (Fig. 5B). 


\section{Discussion}

Growth factor activation of their innate receptors until a restriction point in late $G_{1}$ has been reached is viewed to be necessary for cell cycle progression, whereas ligand-induced growth factor receptor down regulation is perceived to attenuate the growth signal and return the cells to a resting state $[25,26]$. In this study we found, as also shown by others, that EGF-induced $G_{1}$ phase propagation of hepatocytes depended upon continuous EGF receptor activation and signaling through both Erk1/2 and PI3K/Akt pathways $[8,10]$. This signaling activity was necessary even after EGF receptor down regulation and cyclin D induction. Surprisingly, we found that the sustained EGF receptor signaling was not dependent upon continuous EGF stimulation. Instead, EGF induced TGF $\alpha$ release to the medium, albeit at low levels. When TGF $\alpha$ was inhibited by the addition of a neutralizing TGF $\alpha$ antibody to the medium, prolonged EGF receptor signaling was inhibited, induction of cyclin D1 was counteracted, and S phase entry was effectively blocked. Thus, the proliferative effects of EGF appeared to depend on activation of an autocrine TGF $\alpha$ loop.

In this study we have in particular focused on the significance of sustained EGF receptor signaling for hepatocyte proliferation during the phase when the receptor has been downregulated,. In accordance with previous findings, we found that both Erk1/2 and PI3K activities were necessary during this period, as demonstrated by the use of EGF receptor, Mek and PI3K inhibitors. Interestingly, whereas both Erk1 and in particular Erk2 showed conspicuously increased phosphorylation at these time points, Akt phosphorylation during this period was lower than prior to EGF stimulation. The significance of PI3K/Akt activity for cell cycle progression and cyclin D expression is well documented. The D-type cyclins have emerged as main regulators of growth factor-promoted $G_{1}$ progression in mammalian cells $[5,6]$. In hepatocytes, cyclin D1 induces $G_{1}$ progression in response to EGF and insulin through PI3K signaling $[27,28]$. Both Erk1/2 and PI3K/Akt signaling pathways regulate cyclin D1 gene expression. The MAP kinase Erk1/2 induces cyclin D1 gene expression through activation of AP1 and Ets transcription factors [29], whereas PI3K/Akt signaling regulates cyclin D translation through S6K activation and 4EBP1 inactivation, and cyclin D degradation through GSK-3 inhibition [30-33]. In hepatocytes, inhibition of PI3K activity efficiently blocks both cyclin D1 mRNA and protein expression [7]. It is thus intriguing that we found reduced Akt phosphorylation during the period when cyclin D levels increased. When, during this phase, PI3K activity was inhibited by LY294002, cyclin D and proliferation was efficiently inhibited. Thus, reduced, but not abolished PI3K activity allowed cyclin D induction and hepatocyte proliferation. Whether the reduced PI3K activity was necessary for $G_{1}$ progression, or served other functions, remains undetermined. Possibly, our findings implicate that PI3K serves dual functions during cell cycle progression.

Our results determined that sustained EGF receptor signaling and hepatocyte proliferation was dependent on TGF $\alpha$ release to the medium, and that this was induced by EGF stimulation. Inhibition of EGF receptor signaling 12 hours after growth factor addition inhibited proliferation, whereas removal of EGF from the medium at this time point did not. Addition of the anti-TGF $\alpha$ antibody reduced Erk1/2 phosphorylation during this period, which was consistent with our understanding of Erk1/2 functions for hepatocyte proliferation. Akt phosphorylation in EGF-stimulated hepatocytes appeared higher in antiTGF $\alpha$ exposed cells during at least part of the same period. Although this increase in Akt phosphorylation does not intuitively appear consistent with growth inhibition, it should be kept in mind that Akt phosphorylation at these time points was reduced in EGF-stimulated cells compared to unstimulated controls. The neutralizing antibody inhibited cyclin D induction and nuclear translocation of Cdk 4 and Cdk2. This may be a consequence of Erk1/2 inhibition, leading to reduced cyclin D induction and Cdk4 activation, and possibly also of other effects on the intracellular trafficking of G1 cyclins [4, 21]. Addition of the neutralizing antibody to TGF $\alpha$ altered sustained EGF receptor signaling even with EGF present in the medium. This finding suggests that EGF and autocrine TGF $\alpha$ induce dissimilar EGF receptor signals, possibly related to differences in EGF receptor internalization or recirculation. 
Liu et al.: EGF Induces Hepatocyte Proliferation Through Autocrine TGF $\alpha$

It has previously been demonstrated that different growth factors and cytokines activate EGF receptors by transactivating mechanisms. In a hepatocyte cell line, it was shown that $\mathrm{TNF} \alpha$ stimulated proliferation through the release of TGF $\alpha$ to the medium, and that this was achieved through the activation of the metalloproteinase TNF $\alpha$-converting enzyme [14]. Prostaglandin E2 transactivated the EGF receptor and signaling in MH1C1 hepatoma cells but not in primary hepatocytes $[15,34]$. HGF has also been shown to promote liver and hepatocyte proliferation through TGFo induction and EGF receptor transactivation [16]. Transactivation of autocrine TGF $\alpha$ emerges as a principal growth regulatory principle in hepatocytes, and may integrate and modify several growth stimulatory factors. Our demonstration that the effects of EGF receptor activation by EGF depend upon activation of autocrine TGF $\alpha$ introduces a new level of signaling complexity. Since TNF $\alpha$ also has been shown to transactivate EGF receptor through amphiregulin shedding in hepatocellular carcinomas, it is also possible that other EGF receptor ligands (HB-EGF, amphiregulin, betacellulin, epigen, epiregulin) are activated by EGF stimulation[35].

To conclude; our finding that prolonged EGF receptor activation and proliferative signaling depended on stimulation of autocrine TGF $\alpha$ effects identified TGF $\alpha$ transactivation as a major integrating mechanism to achieve cyclin D1 induction and hepatocyte proliferation. Thus, EGF receptor dependency of TGF $\alpha$ may represent a novel mechanism for diversifying growth factor signaling and functional outcome. Further studies will resolve whether such mechanisms are involved in liver regeneration and carcinogenesis.

\section{References}

1 Fausto N, Campbell JS, Riehle KJ: Liver regeneration. Hepatology 2006;43:45-53.

- Taub R: Liver regeneration: From myth to mechanism. Nat Rev Mol Cell Biol 2004;5:836-847.

-3 Moriuchi A, Hirono S, Ido A, Ochiai T, Nakama T, Uto H, Hori T, Hayashi K, Tsubouchi H: Additive and inhibitory effects of simultaneous treatment with growth factors on DNA synthesis through mapk pathway and g1 cyclins in rat hepatocytes. Biochem Biophys Res Commun 2001;280:368-373.

4 Wierod L, Rosseland CM, Lindeman B, Oksvold MP, Grosvik H, Skarpen E, Huitfeldt HS: Cdk2 regulation through pi3k and cdk4 is necessary for cell cycle progression of primary rat hepatocytes. Cell Prolif 2007;40:475-487.

5 Sherr CJ: The pezcoller lecture: Cancer cell cycles revisited. Cancer Res 2000;60:3689-3695.

6 Sherr CJ, Roberts JM: Cdk inhibitors: Positive and negative regulators of g1-phase progression. Genes Dev 1999;13:1501-1512.

7 Coutant A, Rescan C, Gilot D, Loyer P, Guguen-Guillouzo C, Baffet G: Pi3k-frap/mtor pathway is critical for hepatocyte proliferation whereas mek/erk supports both proliferation and survival. Hepatology 2002;36:1079-1088.

-8 Weber JD, Raben DM, Phillips PJ, Baldassare JJ: Sustained activation of extracellular-signal-regulated kinase 1 (erk1) is required for the continued expression of cyclin d1 in g1 phase. Biochem J 1997;326:61-68.

-9 Marshall CJ: Specificity of receptor tyrosine kinase signaling: Transient versus sustained extracellular signal-regulated kinase activation. Cell 1995;80:179-185.

10 Thoresen GH, Guren TK, Christoffersen T: Role of erk, p38 and pi3-kinase in egf receptor-mediated mitogenic signalling in cultured rat hepatocytes: Requirement for sustained erk activation. Cell Physiol Biochem 2003;13:229-238.

11 Thoresen GH, Guren TK, Sandnes D, Peak M, Agius L, Christoffersen T: Response to transforming growth factor alpha (tgfalpha) and epidermal growth factor (egf) in hepatocytes: Lower egf receptor affinity of tgfalpha is associated with more sustained activation of p42/p44 mitogen-activated protein kinase and greater efficacy in stimulation of DNA synthesis. J Cell Physiol 1998;175:10-18.

12 Skov Olsen P, Boesby S, Kirkegaard P, Therkelsen K, Almdal T, Poulsen SS, Nexo E: Influence of epidermal growth factor on liver regeneration after partial hepatectomy in rats. Hepatology 1988;8:992-996.

13 Mead JE, Fausto N: Transforming growth factor alpha may be a physiological regulator of liver regeneration by means of an autocrine mechanism. Proc Natl Acad Sci USA 1989;86:1558-1562. 
Liu et al.: EGF Induces Hepatocyte Proliferation Through Autocrine TGF $\alpha$

14 Argast GM, Campbell JS, Brooling JT, Fausto N: Epidermal growth factor receptor transactivation mediates tumor necrosis factor-induced hepatocyte replication. J Biol Chem 2004;279:34530-34536.

15 Kimura M, Osumi S, Ogihara M: Prostaglandin e(2) (ep(1)) receptor agonist-induced DNA synthesis and proliferation in primary cultures of adult rat hepatocytes: The involvement of tgf-alpha. Endocrinology 2001;142:4428-4440.

16 Tomiya T, Ogata I, Yamaoka M, Yanase M, Inoue Y, Fujiwara K: The mitogenic activity of hepatocyte growth factor on rat hepatocytes is dependent upon endogenous transforming growth factor-alpha. Am J Pathol 2000;157:1693-1701.

17 Sorvillo JM, McCormack ES, Yanez L, Valenzuela D, Reynolds FH, Jr.: Preparation and characterization of monoclonal antibodies specific for human transforming growth factor alpha. Oncogene 1990;5:377-386.

18 Oksvold MP, Skarpen E, Lindeman B, Roos N, Huitfeldt HS: Immunocytochemical localization of shc and activated egf receptor in early endosomes after egf stimulation of hela cells. J Histochem Cytochem 2000;48:21-33.

19 Seglen PO: Preparation of isolated rat liver cells. Methods Cell Biol 1976;13:29-83.

-20 Skarpen E, Lindeman B, Thoresen GH, Guren TK, Oksvold MP, Christoffersen T, Huitfeldt HS: Impaired nuclear accumulation and shortened phosphorylation of erk after growth factor stimulation in cultured hepatocytes from rats exposed to 2-acetylaminofluorene. Mol Carcinog 2000;28:84-96.

21 Wierod L, Rosseland CM, Lindeman B, Oksvold MP, Grosvik H, Skarpen E, Huitfeldt HS: Activation of the p53-p21(cip1) pathway is required for cdk2 activation and s-phase entry in primary rat hepatocytes. Oncogene 2008;27:2763-2771.

22 Brattelid T, Winer LH, Levy FO, Liestøl K, Sejersted OM, Andersson KB: Reference gene alternatives to Gapdh in rodent and human heart failure gene expression studies. BMC Mol Biol 2010;11:22.

-23 Scheving LA, Stevenson MC, Zhang X, Russell WE. Cultured rat hepatocytes upregulate Akt and ERK in an ErbB-2-dependent manner. Am J Physiol Gastrointest Liver Physiol. 2008; 95:G322-331. Sorkin A, Goh LK: Endocytosis and intracellular trafficking of erbbs. Exp Cell Res 2009;315:683-696.

25 Lea NC, Orr SJ, Stoeber K, Williams GH, Lam EW, Ibrahim MA, Mufti GJ, Thomas NS: Commitment point during g0-->g1 that controls entry into the cell cycle. Mol Cell Biol 2003;23:2351-2361.

-26 Zetterberg A, Larsson O, Wiman KG: What is the restriction point? Curr Opin Cell Biol 1995;7:835-842.

-27 Albrecht JH, Rieland BM, Nelsen CJ, Ahonen CL: Regulation of g(1) cyclin-dependent kinases in the liver: Role of nuclear localization and p27 sequestration. Am J Physiol 1999;277:1207-1216.

28 Rickheim DG, Nelsen CJ, Fassett JT, Timchenko NA, Hansen LK, Albrecht JH: Differential regulation of cyclins d1 and d3 in hepatocyte proliferation. Hepatology 2002;36:30-38.

29 Kerkhoff E, Rapp UR: Cell cycle targets of ras/raf signalling. Oncogene 1998;17:1457-1462.

-30 Diehl JA, Cheng M, Roussel MF, Sherr CJ: Glycogen synthase kinase-3beta regulates cyclin d1 proteolysis and subcellular localization. Genes Dev 1998;12:3499-3511.

-31 Goggin MM, Nelsen CJ, Kimball SR, Jefferson LS, Morley SJ, Albrecht JH: Rapamycin-sensitive induction of eukaryotic initiation factor $4 \mathrm{f}$ in regenerating mouse liver. Hepatology 2004;40:537-544.

-32 Hashemolhosseini S, Nagamine Y, Morley SJ, Desrivieres S, Mercep L, Ferrari S: Rapamycin inhibition of the $\mathrm{g} 1$ to $\mathrm{s}$ transition is mediated by effects on cyclin $\mathrm{d} 1 \mathrm{mrna}$ and protein stability. J Biol Chem 1998;273:14424-14429.

-33 Grewe M, Gansauge F, Schmid RM, Adler G, Seufferlein T: Regulation of cell growth and cyclin d1 expression by the constitutively active frap-p70s6k pathway in human pancreatic cancer cells. Cancer Res 1999;59:3581-3587.

-34 Dajani OF, Meisdalen K, Guren TK, Aasrum M, Tveteraas IH, Lilleby P, Thoresen GH, Sandnes D, Christoffersen T: Prostaglandin E2 upregulates egf-stimulated signaling in mitogenic pathways involving akt and erk in hepatocytes. J Cell Physiol 2008;214:371-380.

-35 Berasain C, Nicou A, Garcia-Irigoyen O, Latasa MU, Urtasun R, Elizalde M, Salis F, Perugorría MJ, Prieto J, Recio JA, Corrales FJ, Avila MA: Epidermal growth factor receptor signaling in hepatocellular carcinoma: inflammatory activation and a new intracellular regulatory mechanism. Dig Dis 2012;30:524-31. 